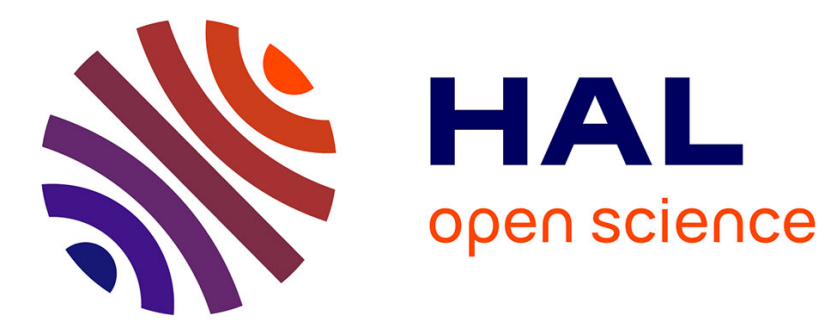

\title{
Sur la vie de Ctésias
}

Bernard A., A. Eck

\section{To cite this version:}

Bernard A., A. Eck. Sur la vie de Ctésias. Revue des Etudes Grecques, 1990, 103, pp.409-434. 10.3406/reg.1990.2487 . hal-01080816

\section{HAL Id: hal-01080816 https://hal.univ-grenoble-alpes.fr/hal-01080816}

Submitted on 17 Mar 2015

HAL is a multi-disciplinary open access archive for the deposit and dissemination of scientific research documents, whether they are published or not. The documents may come from teaching and research institutions in France or abroad, or from public or private research centers.
L'archive ouverte pluridisciplinaire HAL, est destinée au dépôt et à la diffusion de documents scientifiques de niveau recherche, publiés ou non, émanant des établissements d'enseignement et de recherche français ou étrangers, des laboratoires publics ou privés. 


\section{Bernard Eck}

\section{Sur la vie de Ctésias}

In: Revue des Études Grecques, tome 103, fascicule 492-494, Juillet-décembre 1990. pp. 409-434.

\section{Résumé}

Ctésias de Cnide, auteur notamment de Persica et médecin à la cour d'Artaxerxès II, a connu une existence assez mouvementée. Certains épisodes de sa vie sont bien attestés (missions diplomatiques), mais d'autres demeurent obscurs (durée de son séjour en Perse, dernière période de sa vie). Une extrême prudence est donc de mise pour qui veut donner des réponses définitives en ce qui concerne une éventuelle orientation partisane et du personnage et de son œuvre. En tout cas, Ctésias mérite le statut d'historien qu'on lui a trop souvent refusé.

Citer ce document / Cite this document :

Eck Bernard. Sur la vie de Ctésias. In: Revue des Études Grecques, tome 103, fascicule 492-494, Juillet-décembre 1990. pp. 409-434.

doi : $10.3406 /$ reg. 1990.2487

http://www.persee.fr/web/revues/home/prescript/article/reg_0035-2039_1990_num_103_492_2487 


\section{SUR LA VIE DE CTESIAS}

Les différents édite'urs de Ctésias' ${ }^{1}$ et certains historiens ou philologues $^{2}$ se sont efforcés de retracer, tantôt avec prudence tantôt avec une assurance un peu suspecte, tantôt partiellement tantôt presque complètement, la vie de l'auteur des Persica. Je me propose ici de rassembler et de confronter ce qui a été dit, de rappeler parfois des travaux anciens oubliés ou méprisés par les modernes, et d'essayer de donner à un sujet confus et controversé une certaine cohérence. L'entreprise se justifie si l'on songe à la forte originalité du personnage, à la fois historien et médecin, Grec vivant à l'ombre du grand Roi, acteur et

(1) On a consulté H. Stephanus, Ctesiae Cnidii quae exstant opera et fragmenta graece, à la suite de l'édition d'llérodote par A.C. Borheck, 1810, p. $297 \mathrm{sq}$ : J.C.F. Baehr, Ctesiae Cinidii operum reliquiae, 1824, qui accompagne chaque fragment d'un commentaire historique et philologique très complet; C. Müller, Ctesiae Cnidii et chronographorum Castoris, Eratosthenis, etc fragmenta dissertatione et notis illustrata, appendice à l'édition d'Hérodote par Dindorf, 1844, qui reproduit une traduction latine et propose quelques explications et qui ne réédite plus Ctésias dans ses Fragmenta historicorum Graecorum, 1853 - 1870; F. Jacoby, Die Fragmenie der griechischen Historiker III C. n' 688,1958 , dont l'édition, définitive, servira de rèférence; F.W. König, Die Persika des Klesias von Knidos, 1972 (très critiqué) qui, adoptant un principe de classement surprenant (l'ordre alphabétique des auteurs, Photius mis à part), traduit les Persica et commente événement par événement leur contenu. On notera désormais ces ouvrages Estienne, Baehr, Müller, F (ir Hist, König. Je n'ai pas pu disposer de J. Gilmore, The fragments of the Persika of Ctesias, 1888.

(2) H. Ch. M. Rettig, Clesiae Cnidii vita, 1827, peu utilisé et pourtant très riche; C. Lanzani, "I ПEP IIKA di Ctesia, fonte di storia greca ", Rivista di storia antica 5,1,1900, p. 214-231 et 571-602;6,1,1901, p. 66-94;6,2, 1902, p. $316-338$, presque entièrement ignorée, qui s'attache à réhabiliter Ctésias à

R.E.G. tome CIII (1990/2), pp. 409-434. 
témoin de l'histoire; sans aucun doute, avec la vie mouvementée de ce "citoyen du monde" ${ }^{3}$, trait d'union entre deux cultures, nous sommes en présence d'un destin avec lequel celui du seul Xénophon, son contemporain, pourrait rivaliser. Bien plus, retrouver l'homme c'est mieux comprendre les intentions qui se cachent derrière l'œuvre, que nous ne connaissons que par des fragments de seconde main. Ce détour par la vie de Ctésias permettra aussi d'éclaircir un passage obscur du livre II de Diodore de Sicile, dont les chapitres 1 à 34 ont pour source principale Ctésias.

\section{Un médecin au service des Perses.}

Parmi les testimonia ${ }^{4}$ somme toute assez nombreux que nous avons sur Ctésias, les plus riches en renseignements ont été laissés par Plutarque (Vie d'Artaxerxès) et par l'érudit patriar-

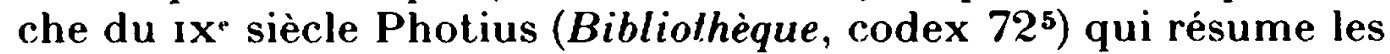
livres 7 à 23 des Persica. Cnidien, fils de Ctésiarchos ou de

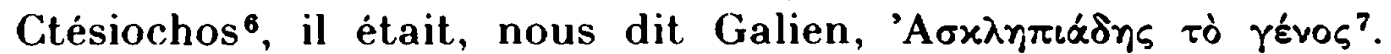
Prisonnier de guerre des Perses, il devient le médecin de cour personnel d'Artaxerxès II Mnémon, s'occupant de la famille et particulièrement de la mère de ce dernier, la terrible et cruelle Parysatis. Cette fonction occupée par Ctésias perpétue probablement une tradition inaugurée par Démokédès, le premier médecin grec appelé à la cour sous Darius $I^{\text {rr }}$ pour remplacer les inamovibles Egyptiens devenus défaillants ${ }^{8}$.

travers son récit des guerres médiques, du soulèvement d'Egypte, de l'expédition de Cyrus le Jeune et des rapports entre Evagoras et la Perse et qui le confronte aux autres sources littéraires grecques; F. Jacoby, "Ktesias». $\boldsymbol{R} \boldsymbol{F}$ XI, 2, 1922, col. 2032-2073, parfois catégorique; T.S. Brown, "Suggestions for a vita of Ctesias of Cnidus ", Iistoria 27, 1978, p. 1-19, dont les propositions sont parfois brillantes. On notera désormais ces études Rettig, Lanzani, Jacoby, Brown. Je n'ai pas pu me procurer l'ouvrage de l'historien russe $J$. W. Pjankow (Klesias, Duschanbe, 1975). On consultera aussi l'ouvrage récent de J. Boncquet, Diodorus Siculus (II, 1-34) over Mesopolamië, Een historische kommentaar, 1987, qui consacre quelques développements à Ctésias (voir notamment p. 200-204).

(3) Expression de Lanzani, p. 335.

(4) Voir F GR Hist, p. 416-420, T1-7 (vie) et T8-19 (auvres).

(5) Voté désormais simplement Photius.

(6) F Gr Hist, T1.

(7) F Gr IIist, T4; Rettig, p. 6-7, commente l'expression.

(8) C. Hérodote, III, 129 - 133 : Démokédès guérit la cheville déboîtée de Darius puis l'abcès au sein d'Atossa. Baehr, p. 16-17, et Lanzani, p. 219, relèvent le parallèle; sur les Grecs au service du Grand Roi, voir O. Picard, Les 
Ctésias fait d'ailleurs mention d'un autre médecin grec, sous Artaxerxès $I^{\circ r}$, Apollonidès de Cos, mais le comportement qu'il lui prête - il devient l'amant d'Amytis, la fille du roi - et le sort qu'il subit - il est enterré vivant ${ }^{9}$ - rendent son témoignage, attesté nulle part ailleurs, suspect; il me semble que Ctésias discrillite celui qui fut peut-ètre son prédécesseur direct ou que son ricit se fait l'écho de la rivalité entre l'école de Cnide et celle de ciosio.

Personne ne cont'ste que, en tant que médecin, Citésias était un proche du roi, parfaitement intégré dans le petit monde des courtisans; le parfum de scandale familial qui se dégage des Persica, les intrigues de sérail, les manigances des eunuques prouvent suffisamment sa connaissance de la cour perse. Sans

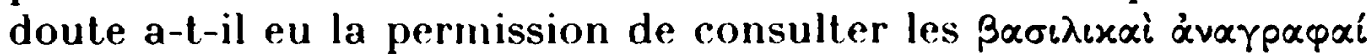

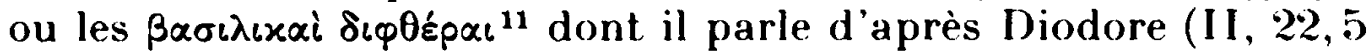
et 32,4$)$; reste à démontrer solidement qu'il a effectivement utilisé ces archives royales ${ }^{12}$ qu il a peut-ètre survolées par curiosité, sans ètre animé par une intention d'historien, et dont

Grecs devant la menace perse. 1980, p. 51-64, I. M. I.ewis, Sparta and Persia, 1977, p. 12-14, et G. Walser. Hellas und Iran, 1984, p. 20-26.

(9) F Gr Hist, F14= Photius, 41 b 8-23.

(10) Pour une vue traditionnelle (que l'auteur réfute) concernant les divergences et les principes de l'opposition entre ces deux écoles, auxquelles il faut rajouter celle de Sicile, voir A. Thivel, Cnide et Cos? Essai sur les doclrines médicales dans la collection hippocratique, 1981, p. 7 et 53-58; l'ensemble de son étude remet complètement en cause cette distinction et les critères qui la fondent.

On ne sait rien des théories médicales de Ctésias; cependant. comme les médecins grecs devaient connaitre la médecine indienne par la Perse, Ctésias a peut-ètre servi d'intermédiaire (A. Thivel, p. 321-322). D'ailleurs, il avait certainement une bonne connaissance de l'Inde et il reste d'importants fragments de ses Indica (F Gr Hist, F45 à F52). Sur la médecine, seuls deux fragments assez insignifiants subsistent ( $F$ Gr Hist, F67 et F68) dont l'un (F68 = Oribase, Collect. med. 8,8) porte sur l'emploi de l'ellébore (cf. commentaire d'A. Thivel, p. 90-91).

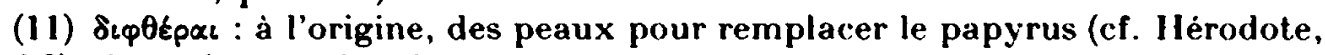
$\mathrm{V}, 58$ ), donc des parchemins.

(12) Oui pour Baehr, p. 17-18; Müller, p. 3 ("Ctesias e Persicis fontibus sua depromens omnia»); König p. 33. Mais Rettig, sceptique, remarque justement que cet usage des archives royales aurait signifiè qu'il ait confiè à Artaxerxès son projet d'écrire une histoire (p. 16). Pour R. Drews, comme la durée que Cásias assigne à l'empire assyrien ("plus de 1300 ans", Diodore, II, 28,8 ou "plus de 1360 ans ", 21,8) ainsi que les trente rois (Diodore, II, 21, 8) correspondent à peu près à la réalité, l'utilisation par Ctésias des archives royales est presque certaine (*Assyria in Classical Universal Histories", Historia 14, 1965, p. 138-42). 
il s'est souvenu bien plus tard ${ }^{13}$. Au demeurant, il est parfaitement légitime de mettre en doute l'existence même de ces archives et d'y voir des documents fictifs dont personne ne peut vérifier l'authenticité; ces annales imaginaires masqueraient, en réalité, soit une information orale provenant, au

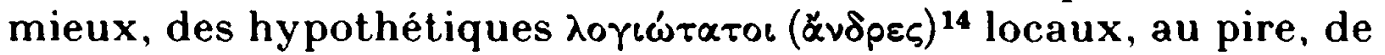
racontars de courtisans, soit une information livresque d'origine grecque (Hérodote), soit enfin la propre fantaisie de Ctésias ${ }^{15}$. Sans vouloir examiner ici la question complexe des sources de Ctésias, on se contentera de la formuler en termes clairs ${ }^{16}$ : sontelles orales ou écrites? perses ou grecques? surtout, Ctésias est-il étroitement dépendant ${ }^{17}$ ou au contraire totalement indépendant $^{18}$ d'Hérodote, dont les Histoires recoupent en partie les

(13) Lanzani, p. 335-336.

(14) Diodore, II, 4,3.

(15) Cette contestation des sources perses est l'œuvre de Jacoby, col. 2047 2049, qui dénonce avec une bonne argumentation les méthodes de la "PseudoI Iistorie" employées par Ctésias (avis que partage Brown, p. 12, n. 52).

(16) Comme le fait P. Krumbholz, qui penche pour une information orale : "Welches waren aber die Quellen des Ktesias? mündliche oder schriftliche? poetische oder prosaische?" ("Zu den Assyriaka des Ktesias", Rh. Mus. 52, 1897. p. 282).

(17) Pour Jacoby, Ctèsias copie Hérodote tout en polémiquant contre lui; ainsi, col. 2053, la Sémiramis de Diodore II n'est qu'un concentré purement littéraire des deux reines Sémiramis et Nitokris dont parle Hérodote, avec l'addition de traits d'Atossa telle que l'évoque Hellanikos. Sur ce point. Jacoby a tort (sur l'indiscutable noyau historique du personnage, voir W. Eilers, Semiramis, Entstehung und Nachhall einer allorienlalischen Sage, Oster. Akad. der Wiss. 274, 2, 1971, p. 34-38; sur l'ancienneté et le syncrétisme de la légende, liée à l'Iran et à l'Arménie, ibid., p. 25-33). J. M. Bigwood relève, pour une autre période certes, des similitudes entre les deux historiens, mais pour lui Ctésias a surtout la volonté de corriger Hérodote, son rival, fut-ce aux dépens de la vérité ("Ctesias as historian of the Persian Wars", Phoenix 32, 1978, p. 22-24).

(18) Lanzani, dans toute son étude, s'attache à voir dans les récits d'Jérodote et de Ctésias deux versions historiques distinctes et crédibles, de mème valeur; elle conclut, p. 337, avec la certitude que Ctésias ne s'est pas servi d'llérodote bien qu'il ait eu son cuvre entre les mains, reconnaissant à Ctésias une meilleure connaissance de la Perse et une certaine rivalitè à l'encontre d'Hérodote.

On ne peut sans doute ni surestimer l'influence d'llérodote, ni l'éliminer radicalement. En vérité, il semblerait que tout dépende du segment historique qui fait l'objet d'une narration. Ainsi, dans le récit des origines de Cyrus, A. Momigliano ("Tradizione e invenzione in Ctesia". Quarto contributo alla storia degli studi classici e del mondo antico, Storia e lelteratura 115, 1969, p. 193-200. article repris d'Alene e Roma $2,12,1931$ ) note à la fois des emprunts à llérodote et les apports d'une tradition orientale. 
Persica? L'examen de l'onomastique du livre 1I, 1-34, de Diodore ou de calle du codex 72 de la Bibliolhèque de Photius et la comparaison avec l'onomastique perse que l'on découvre, par exemple, sur les tablettes de Persépolis pourraient, le cas échéant, à la lumière de certains travaux ${ }^{19}$, déterminer partiellement l'apport perie dans l'information de Ctésias.

Mais revenons a sa fonction de médecin dans l'entourage intime du roi pour imaginer que vraisemblablement, comme Démokèdès, il avait un oixos $\mu \varepsilon^{\prime} \iota \tau_{0 \varsigma}{ }^{20}$ et qu'il était un

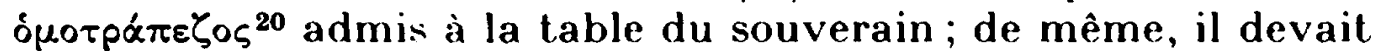
parler perse ${ }^{21}$-.- c'est évidemment invérifiable - et, surtout, il est sans doute le seul historien grec à ètre dans ce cas ${ }^{22}$. Il suivait aussi la cour dans ses déplacements vers les différentes capitales de l'empire et, à propos de la description de Babylone au livre II de Diodore (chap. 7 à 10), il est important de savoir si Ctésias avait vu Babylone. Or, Artaxerxès II, qui ne semblait pas apprécier cette ville ${ }^{23}$ malgré sa position centrale dans l'empire, y séjourne dans son palais d'hiver en $395^{24}$, date à laquelle Ctésias est déjà retourné en Grèce (cf. infra); et, auparavant, en 400 , s'il a exilé dans sa ville natale sa mère coupable d'avoir empoisonnè sa femme Stateira ${ }^{25}$, c'est dans la ferme intention de ne plus revoir ni sa mère ni Babylone. Ainsi, on a la certitude d'un seul séjour de Ctésias à Babylone ${ }^{26}$, à

(19) Fintre autres, ceux d'E. Benvéniste. Titres el noms propres en iranien ancien, 1966; (i. Cardascia, Les archives de Murashu, 1951; L.Zgusta, Kleinasialische Personennamen, 1964; H. Schmitt, "Medisches und persisches Sprachgut bei llerodot ". Zeitschrift der Deutschen Morgenländischen Gesellschafl 117,1967 . p. 119-144: M. Mayrhofer, Onomastica persepolitana, Oster. Akad. der Wiss. 286, 1, 1973. Voir aussi les études rassemblées par $R$. Drews, art. cit., p. 139, n. 43 à 46.

(20) II Ṕrodote, III, 132.

(21) "... ut linguam et instituta barbarorum penitus cognoscere posset" (Müller, p. 3).

(22) O. Picard, Les Grecs devanl la menace perse, 1980, p. 202.

(23) II restaure à grands frais Suse, Ecbatane et, sur la fin de son règne, Persépolis sans rien construire à Babylone (cf. A. T. Olmstead, History of the Persian Empire, 1948, p. 422-424).

(24) C.f. IDiodore, XIV, 81, 4-5 (Conon vient l'y trouver).

(25) F Gr Ilist, F29, b = Plutarque, Artaxerxès 19. "Il la relégua, consentante, a Babylone, en disant que tant qu'elle vivrait, lui-même ne reverrait pas Babylone" (trad. R. Flacelière et F. Chambry, (CU F, 1979).

(26) Voir J. M. Bigwood, "Ctesias" description of Babylon ", American .Journal of Ancient Ilistory 3,1978, p. 32-33. Mais on verra plus loin la possibilité d'un deuxième séjour à Babylone. 
savoir dans les lendemains de la bataille de Counaxa, proche de Babylone où Artaxerxès blessé s'était retiré et où le mercenaire Cléarque fut emprisonné un peu plus tard ${ }^{27}$. Cela suffit pour affirmer que Ctésias connaissait la ville.

C'est précisément à Counaxa (septembre 401) que notre médecin, à qui nous devons de connaître le nom de l'endroit ${ }^{28}$, s'illustre : quand le javelot de Cyrus blesse son frère Artaxerxès qui tombe de cheval, Ctésias et d'autres l'accompagnent à l'écart du combat ${ }^{29}$; là, Ctésias soigne son protecteur ${ }^{30}$ qui le comble ensuite d'honneurs ${ }^{31}$. Or, Xénophon se trouvait dans le camp opposé et son œuvre ne mentionne que deux fois Ctésias qu'il présente comme un écrivain et non pas comme quelqu'un qu'il aurait vu ${ }^{32}$; l'authenticité de ces deux mentions a du reste été contestée ${ }^{33}$, peut-être à tort car tous les éditeurs les admettent. Il semble que Xénophon, qui n'a pas dû voir la charge de Cyrus et des siens puisque les Grecs combattaient dans un autre secteur ${ }^{34}$, s'est en partie inspiré par la suite de Ctésias, témoin oculaire du duel entre les deux frères ${ }^{35}$; de plus, ce duel est rapporté de façon assez similaire par Plutarque ${ }^{36}$ qui suit de son propre aveu Ctésias. Il paraît donc probable que Xénophon n'a ni vu ni connu Ctésias, si peu mentionné par lui, mais qu'il a lu son cuvre bien après les événements et que ${ }^{37}$, sans l'avoir sous les yeux, il s'est rappelé certains passages pour rédiger l'Anabase, malgré une mémoire peut-être un peu défaillante ${ }^{38}$.

(27) F Gr Hist, F27, 69= Photius, 44 a 29-34.

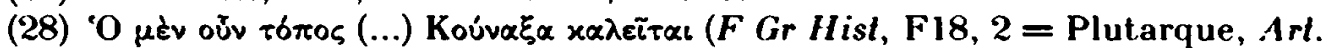
$8,2)$. Xénophon ne fait nulle part mention du nom de Counaxa.

(29) F Gr Hist, F20, 2-3 = Plutarque, Art. 11, 2-3; cf. aussi Diodore, XIV, 23,6, mais sans évocation de Ctésias, car Diodore ne s'inspire de Ctésias qu'à travers Ephore, source du livre XIV (voir Lanzani, p. 331, et E. Schwartz, "Diodoros", $R E \mathrm{~V}, 1905$, col. 680).

(30) F Gr Hist, F21, 26=Xénophon, Anabase 1, 8, 26. Le silence de Plutarque sur ce qui est certes une évidence est néanmoins un exemple de sa malveillance à l'égard de Ctésias.

(31) F Gr Hist, F26, 1 = Plutarque, Art. 14, 1.

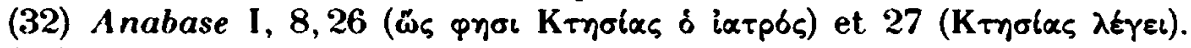

(33) Voir, chez Lanzani, la note 1 p. 217.

(34) Anabase I, 8, 18-20.

(35) Anabase I, 8,21,27.

(36) Art. 11; F. Jacoby (F Gr Hist) a raison de placer còte à côte les deux passages (F20 et F21).

(37) Lanzani, p. 318.

(38) Sinon, il aurait tout de mème mentionné le nom de Counaxa. 


\section{Un partisan di: Sparte? de la Perse?}

La médecine n'a pas été le seul centre d'intérêt de Ctésias. Sans vouloir examiner ici sa valeur en tant qu'historien, il convient toutefois d'essayer de savoir s'il était animé d'intentions partisanes soit dans sa vie, soit dans son ouvre. Photius note qu'il "fit beaucoup (...) pour soulager et soigner Cléarque dans sa prison " ${ }^{39}$, le mercenaire spartiate de Cyrus capturé peu après Counaxa par le dévoué Tissapherne ${ }^{40}$; et Plutarque, dont l'opinion a beaucoup compté dans l'appréciation de Ctésias, nous dit qu'il vantait constamment les mérites de Sparte ${ }^{41}$. Ainsi, en ces années troubles où l'on vit les derniers soubresauts de la guerre du Péloponnèse, Ctésias aurait pris une position politique pro-spartiate et il aurait confirmé par la suite, dans ses Persica, sa laconophilie ${ }^{42}$.

Une telle affirmation semble exagérée. En effet, à considérer ce que l'on sait de sa vie, force est de constater la maigreur des indices. Cnide fait certes partie, à une période donnée, de la zone d'influence spartiate ${ }^{43}$, mais on chercherait en vain chez Ctésias une trace de patriotisme ${ }^{44}$ ou d'attachement affectif à Cnide ${ }^{45}$.

(39) 44 a. 1. 32-34. trad. R. Henry, coll. byz.. 1959; cf. aussi F (ir Hist. F28, 1-4 = Plut.. Arl. 18, 1-4.

(40) Anabase II, 5,30-32. Sur Cléarque, fasciné par la guerre et le commandement militaire, voir le portrait qu'en fait Xénophon, Inab. II. 6, 1 15 : l'adjectif $\varphi$

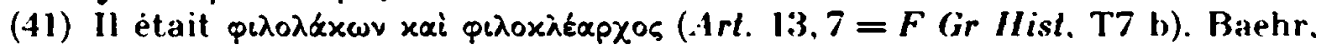
très à l'opposé de l'ensemble de la critique, défend Ctésias contre Plutarque, trop imprégné de préjugés, avec un certain humour : At erat $\phi \iota \lambda o \lambda \alpha x \omega v$. Fuerit

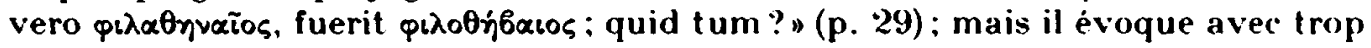
d'optimisme sa $\varphi i \lambda \alpha \lambda \eta \theta \varepsilon i \alpha$ (p. 31).

(42) Leitmotiv de Jacoby (par exemple dans le récit des guerres médiques, col. 2061). J. M. Bigwood (Ctesias as historian of the persian wars", Phoenix 32, 1978 , p. 19-41) est plus nuancé : ainsi, le chiffre ridicule de 300 avancé par Ctésias pour le contingent spartiate à Platées n'est pas à la gloire de Sparte (p. 28-31). Relevons au passage la grossière bévue de Ctésias pour qui Platées précède chronologiquement Salamine (F Gr Hist, F13, 28-30 = Photius, 39 a 40 41 et 39 b 1-27) et ses graves oublis (Artémision, Mycale).

(43) J. M. Bigwood (art. cit., p. 29) note que Cnide s'ètait détachée d'Athènes en $412 / 11$ et qu'elle était restée jusqu'en 394 une importante base navale spartiate. Rajoutons que, d'après Hérodote, I, 174, Cnide était une colonie de Sparte.

(44) Lanzani, p. 335.

(45) Pourtant, à en croire Jacoby, col. 2035 , il ne manque pas une occasion de parler de lui et de tout ce qui le touche directement. 
Quant à son amitié pour Cléarque, elle ne signifie pas nécessairement une approbation de la politique spartiate et

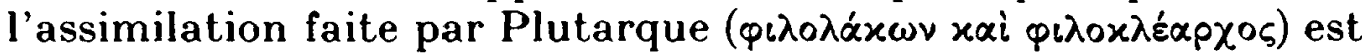
peut-être trompeuse ; bien au contraire, il est permis d'imaginer une hostilité de Sparte à l'égard de Cléarque puisqu'on n'a guère souligné que les Spartiates le condamnèrent à mort par contumace pour sa désobéissance en Thrace ${ }^{46}$. Je verrais plus volontiers en Cléarque, à la lumière des textes, un de ces nombreux condottieri spartiates menant une politique personnelle aventureuse mal définie par rapport à celle de la métropole; que Ctésias l'ait réconforté dans sa captivité pourrait s'expliquer par la peur que lui inspirait Parysatis : il lui fallait gagner la sympathie de la redoutable reine-mère qui favorisait Cléarque ${ }^{47}$, le compagnon d'armes de son cher Cyrus qu'elle avait toujours préféré à Artaxerxès ${ }^{48}$. Ce dernier, par contre, détestait les Spartiates ${ }^{49}$, se démarquant ainsi de ses prédécesseurs sur le trône ${ }^{50}$; aussi Ctésias n'a-t-il pas dû faire part à son maître de son éventuelle laconophilie, à supposer que ce sentiment ait pris naissance déjà en Perse. La période finale de la vie de Ctésias, examinée par la suite, loin d'éclaircir la question de son appartenance politique, ne fait que l'obscurcir davantage.

Lire minutieusement presque l'ensemble de l'œuvre sous cet angle permettrait de trouver une solution; seule Lanzani s'y est attachée, comparant surtout le récit des guerres médiques fait par Ctésias aux témoignages d'Hérodote et de Thucydide notamment ${ }^{51}$. Ses conclusions révèlent que Ctésias suit une source et une tradition spartiates ${ }^{52}$ l'amenant à glorifier Sparte mais sans qu'il en soit un partisan avoué ; rapportant le point de vue de Sparte simplement parce que c'est la cité la plus puissante en Grèce après la guerre du Péloponnèse, Ctésias ne

(46) Xénophon, Anabase II, 6,2-4. Sur ses actes tyranniques commis à Byzance et sur son ralliement à Cyrus en 403, voir Diodore, XIV, 12,3-9; mais Xénophon ne le noircit pas à l'occasion du siège de Byzance (Helléniques I, 3, 1519).

(47) Voir Plutarque, Arl. 18, 3,5-6.

(48) Voir, par exemple, Xènophon, Anabase I, 1, 4 ou Plutarque, Art. 2, 3.

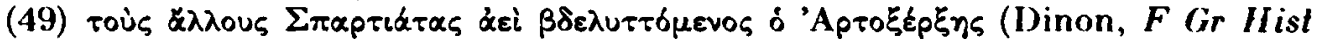
n" 690, F19 = Plutarque, Art. 22,1).

(50) Voir D. M. Lewis, Sparta and Persia, 1977, p. 25-26.

(51) P. 224-230; 571-602; 66-77.

(52) Peut-ètre une source perse pour la bataille de Marathon (p. 230) 
fait que refléter en toute bonne foi une idéologie dominante sans avoir lui-même de couleur politique précise ${ }^{53}$. De plus, faire de Ctésias un partisan de Sparte supposerait qu'il manifeste une tendance anti-athénienne constante; or, ce point est loin d'être établi, exemples et contre-exemples s'opposant ${ }^{54}$.

Par ailleurs, il convient de s'interroger sur la nature des sentiments de Ctésias à l'égard du pouvoir perse, préliminaire indispensable pour la compréhension d'une partie du livre II de Diodore (chap. 1 à 34) ${ }^{55}$. L'imbroglio est complet et n'est pas près d'être démêlé : Ctésias aurait été un porte-parole achéménide $^{56}$ ou au contraire un discret opposant ${ }^{57}$ ou enfin un personnage assez neutre ${ }^{58}$. On se contentera ici de cerner les données de cette question délicate. Rappelons tout d'abord la traditionnelle division des Persica, postérieure à Ctésias, en Assyriaca (livres I à VI) et en Persica proprement dits (livres VII à XXIII), compilés principalement mais pas exclusivement les uns par Diodore, les autres par Photius ${ }^{59}$. Deux types d'événements bien distincts, sur lesquels Ctésias ne peut

(53) P. 335-337.

(54) Ainsi, aux 40 vaisseaux athéniens envoyés en Égypte à Inaros alors que Thucydide en mentionne 200 (cf. J. M. Bigwood, "Ctesias'account of the revolt of Inarus", Phoenix 30, 1976) répondent l'évocation de Miltiade (F Gr Hist, F13. 22), celle de Themistocle et relle d'Aristide (F13, 30), sans parler de l'affaire Conon (cf. infra).

(55) Correspondant à peu de choses près à $F$ Gr llist, F1 b et F5.

(56) Pour G. Goossens, "L'histoire d'Assyrie de Ctésias". Ant. Class. 9, 1940 ("On peut supposer que dans ses ouvrages il a rapporté tout naturellement le point de vue officiel sur l'histoire de l'empire perse et des empires qui l'avaient précédé", p. 26).

(57) Pour M. Braun, History and Bomance in Graeco-Oriental Literature, p. 69, des intentions hostiles aux Perses se lisent dans l'histoire de Ninos et de Sémiramis (cité par G. Goossens, art. cit., p. 27, n. 8).

(58) Pour Lanzani, p. 336, Ctésias, esprit impartial, n’a pas voulu glorifier les Perses ; pour J.M. Bigwood, "Ctesias was not writing pro-Persian propaganda" ("Ctesias' account of the revolt of Inarus", Phoenix 30, 1976, p. 16). Toutefois. dans ces deux études, l'histoire d'Assyrie n'est pas envisagée.

(59) Pour la répartition détaillée des 23 livres des Persica, voir Jacoby. col. 2040-2044 et König, p. 28-34 (leurs divergences sont minimes). Müller intitule MHAIKA les livres IV à VI, mais il s'agit là pour Jacoby et König d'une subdvision des Assyriaca; chez Diodore, qui résume fortement cette section médique, le passage de l'histoire assyrienne à l'histoire médique s'effectue en II. 32,5, quand Arbace a détrôné Sardanapale (pour Müller et Jacoby) ou peut-ètre déjà en II, 23,1, quand Sardanapale entre en scène (pour König). 
pas porter le même regard, sont donc rapportés : d'une part une proto-histoire perse très étendue dans le temps et plus ou moins mythique (Ninos, Sémiramis, l'Assyrie), d'autre part une histoire plus proche (de Cyrus le Grand à Artaxerxès II) qui peu à peu rejoint son propre vécu et l'implique ${ }^{60}$. On constate que l'information de Ctésias est à nouveau en jeu; ainsi, pour ce qui est des Assyriaca, on ne voit pas comment il a pu ne pas utiliser en partie des sources littéraires, grecques ou perses; quant aux sources des Persica (VII-XXIII), la question a été posée plus haut ${ }^{61}$. En tout cas, utiliser des sources perses, s'il n'y en a pas d'autres, ne signifie pas nécessairement faire œuvre de propagande.

L'examen du rapport entre les deux sections des Persica ne nous renseigne pas davantage sur les intentions de son auteur car diverses interprétations coexistent. On peut voir en effet, des Assyriaca aux Persica proprement dits, une continuité sans heurt, une passation de pouvoirs entre les peuples ${ }^{62}$, ou au contraire une cassure, une rupture, l'histoire de l'Assyrie étant celle d'une décadence à laquelle les Mèdes puis les Perses mettent fin $^{63}$. Tout dépend, il est vrai, si l'on met l'accent sur la geste héroïque de Ninos et de Sémiramis (Diod., I I, 1-20) ou sur les errements de Ninyas et de Sardanapale (Diod., II, 21-32). II est mème permis de lire, après l'examen par exemple de la liste des conquètes de Ninos (Diod., II, 2,3), une récupération rétrospective de l'histoire de l'Assyrie au profit du pouvoir achéménide ${ }^{64}$. Néanmoins, pareilles vues plaident plus ou moins pour une sympathie de Ctésias à l'égard des Perses.

(60) Jacoby, pourtant si sévère envers Ctésias, reconnaît que plus le récit se rapproche de sa propre époque, plus les renseignements sur l'état intérieur de la Perse deviennent dètaillés (col. 2059).

(61) C.f. supra p. 412-413.

(62) Jacoby, col. 2041 ; une des rares originalités de Citésias par rapport à IIérodote qui marque très distinctement les trois coupures dans l'histoire de l'Orient et leurs contrastes (Assyriens, Mèdes, Perses cf. I, 95 sq.).

(6:3) P. Krumbholz, "Zu den Assyriaka des Ktesias", Rh. Mus. 52, 1897. p. 281

(64) I. Borzsák rapproche cette liste de celle des conquêtes de Cyrus et constate que les Perses amplifient et sapproprient le passé historique assyrien, fondant et légitimant ainsi, par une manouve de propagande, leur propre imperialisme ("Semiramis in Zentralasien", Acla antiqua .1cademiae Scientiarum Ilungaricae 2.4, 1976, p. 53-55). Mais il ne dit pas explicitement qu'il s'agit là d'une volonté délibérée de la part de Citésias. König, p. 35-36. compare cette mème liste à celle des conquètes de I larius Ier, mais il assimile la majeure partie de l'o'uvre de Ninos à celle de Sarrukinu II, qui régna de 721 à 705 . 
Mais une fois qu'intervient, avec les guerres médiques, le monde grec, cette sympathie ne doit-elle pas être remise en cause? Qu'en est-il alors de sa prétendue laconophilie? Comment concilier, en effet, ici une tendance pro-spartiate, là une tendance pro-achéménide, si ce n'est en déniant à Ctésias cette indispensable cohérence qu'on est en droit de poser comme un a priori? La position de Ctésias face aux Perses semble donc difficile à déterminer et reste à mes yeux problématique; j'ajouterais que l'intérêt qu'il pouvait avoir à devenir le chantre des rois perses n'apparaît pas puisqu'on verra qu'il a vraisemblablement rédigé et publié les Persica après avoir quitté définitivement la Perse. Enfin, de toute évidence, la question de l'appartenance politique de Ctésias devient insoluble, voire absurde, s'il s'avère que les fragments que nous possédons ont été partiellement remaniés par des intermédiaires ${ }^{65}$. Dans ce domaine, les seules et maigres certitudes portent sur son opportunisme très poussé ${ }^{66}$ au service d'un individualisme ${ }^{67}$ assez éloigné d'un quelconque idéal nationaliste. Mais laissons à nouveau parler les faits.

(65) Ainsi, à propos du seul passage de la liste des conquètes de Ninos (IDiod., II, 2,3), Th. Nöldeke doute qu'il reproduise exactement celui de Catésias

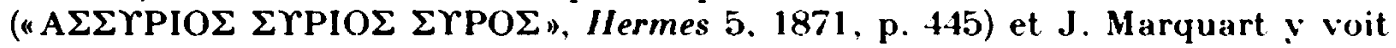
les traces d'Agatharchidès ("Die Assyriaka des Ktesias". Philologus Supplementband 6, 1891-93, p. 522). On a cru déceler aussi, entre Ctésias et Diodore II, Clitarque ou Hiéronymos; J.M. Bigwood donne une liste quasi exhaustive de ceux qui penchent pour un intermédiaire, de ceux qui voient dans le livre II de Diodore (1-34) un emprunt direct à Ctésias, et même des incertains ("Diodorus and Ctesias", Phoenix 34, 1980, p. 196, n. 5): pour lui, Diodore a lu Ctésias dans le texte, malgré quelques apports étrangers (cf. déjà Baehr, p. 388).

Il est vrai qu'il est tentant de comparer le couple Ninos-Sémiramis à Alexandre et que, comme le souligne I. Borzsák (art. cit., p. 56), il est difficile de séparer nettement, chez. Diodore, ce qui provient de Ctésias et les traces laissées par les historiens d'Alexandre (dont Clitarque), ou de savoir si Sémiramis est influencée par Alexandre ou si c'est l'inverse qui se produit ; en tout cas, d'après lui (p. 58-60), Diodore est saisi par l’imitalio Alexandri dans son récit concernant le hèros égyptien Sésostris (I, $53 \mathrm{sq}$.). On a d'ailleurs rapproché, mais allusivement, Ninos de Sésostris (pour Jacoby, col. 2042, Ninos est un pastiche dènaturé - "Abklatsch" - de Sésostris) et aussi Sémiramis de Sésostris (cf. A. Burton, Diodorus Siculus - Book I - A commentary, 1972, p. 175, qui remarque que Ctésias et. Sémiramis sont cités en $I, 56,5)$; un examen plus précis des textes semble toutefois nécessaire avant de pouvoir affirmer tout cela.

(66) Suffisamment prouvé par ses accointances avec l'odieuse mais influente Parysatis.

(67) Constamment souligné par Jacoby. 


\section{Citésias diplomate.}

En deux circonstances différentes, il semble que Ctésias a été chargé par les Perses d'une mission à caractère diplomatique auprès des Grecs; sa participation est vraisemblable dans le premier cas, quasi certaine dans le second, et, à nouveau, elle pourrait bien s'incrire dans une tradition puisque le médecin Démokédès déjà avait été utilisé d'une manière similaire ${ }^{68}$. Sans préjuger d'une connivence politique des médecins grecs avec leurs maîtres perses et sans vouloir surestimer leur intelligence, on imaginera simplement qu'ils ont servi d'interprètes ${ }^{69}$.

Ctésias aurait été de ceux qu'Artaxerxès envoya après Counaxa auprès des mercenaires grecs, et notamment auprès de Cléarque, pour les désarmer, et cela sans succès d'ailleurs. C'est lui-même qui le rapporte, d'après Plutarque, mais celui-ci réfute aussitôt son témoignage en le qualifiant de $\lambda \alpha \mu \pi p o ̀ v ~ \psi \varepsilon \tilde{\sigma} \sigma \mu \alpha$ parce que Xénophon ne mentionne pas sa présence ${ }^{70}$. Il est vrai que le récit de Xénophon ${ }^{71}$, sans exclure d'autres Grecs ${ }^{72}$, ne nomme que le seul Phalinos ${ }^{73}$, au rôle primordial; mais nous avons vu plus haut que Xénophon ne connaissait probablement pas Ctésias. On a beaucoup glosé sur cette ommission : ainsi, Xénophon n'aurait pas remarqué Ctésias, personnage muet de la scène, confondu avec les barbares ${ }^{74}$; ou encore, passer sous silence la présence de Ctésias serait pour Xénophon une manière de réfuter son récit et d'en souligner le caractère mensonger ${ }^{35}$;

(68) Il avait servi de guide aux Perses au cours de la première mission d'exploration du monde grec; il en avait profité pour rejoindre Crotone. sa patrie, mais il fut repris (Hérodote, III, 135-138).

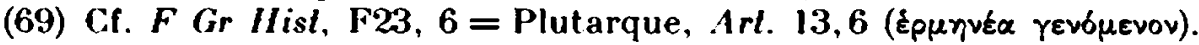

(70) F Gr Hist, F23, 5-6= Plutarque, Art. 13,5-6. Plutarque sait que Xénophon avait lu Ctésias, mais on ne peut pas déduire clairement de ce passage que Plutarque croit qu'il l'a connu en Perse.

(71) Anabase II, 1,7-23 (cf. aussi Diodore, XIV, 25, 1-6; sur Diodore XIV, voir supra p. 414, n. 29).

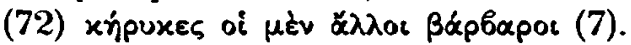

(73) Phalynos chez Diodore, Phaullos dans F Gr Hist, Phallynos chez. Plutarque (voir apparat critique C.U.F., 1979).

(74) Rettig, p. 20, mais il repousse cette hypothèse; de mème, Lanzani, p. 318, pense que Phalinos était le véritable représentant du Grand Roi et que Citésias passa inaperçu.

(75) Rettig, p. 21, s'inclinant ainsi devant l'autorité de Xénophon. Inversement, Lanzani, p. 318, estime que s'il avait voulu contester Ctésias, il l'aurait fait explicitement. 
ces hypothèses que rien ne peut ètayer me paraissent gratuites. De même, on a suggéré, pour concilier la version de Xénophon et celle de Citésias-Plutarque, quelles évoqueraient deux moments différents ${ }^{76}$; Ctésias aurait donc fait partie de la deuxième ambassade ${ }^{77}$ qui vint le lendemain de la première proposer une trêve aux Grecs ${ }^{78}$. Mais les textes ne mentionnent pas la présence de Phalinos ni celle d'aucun Grec d'ailleurs, alors que Ciésias précise bien qu'il était avec Phalinos; or, comme Phalinos n'est mentionné que dans la première ambassade, c'est. de celle-ci seulement qu'il doit être question.

On est finalement réduit à trancher entre les dires de Ctésias et ceux de Plutarque, qui les nient. Connaissant la malveillance de Plutarque à l’égard de Ctésias, on peut donc supposer que ce dernier a fait partie, comme il le dit, des négociateurs venus réclamer aux Grecs leurs armes après Counaxa ${ }^{79}$.

Mais, surtout, il est bien attesté que Ctésias prit une part active dans une operation de plus grande envergure; il joua en effet un rôle important dans les tractations qui aboutirent à l'engagement comme amiral au service du Grand Roi de J'Athénien Conon ${ }^{80}$, le vaincu d'Aigos Potamos (405) qui, plus tard, allait détruire devant... Cnide (394) la flotte spartiate autrefois alliée aux Perses. Plutarque ${ }^{81}$ et surtout Photius ${ }^{82}$ mettent en avant avec quelques variantes la figure de Ctésias ${ }^{83}$. A partir de $399^{84}$, celui-ci remplit une délicate fonction d'intermédiaire dans un chassé-croisé épistolaire assez confus entre les différents partis, allant mème jusqu'à porter en personne des lettres confidentielles d'une part à Conon ${ }^{85}$, réfugié

(76) Intuition de Rettig, p. 21.

(77) Iypothèse proposée par L. Holzapfel dans son compte rendu des articles de Lanzani (Berliner Philologische Wochenschrift 25, 1905, col. 1269 ).

(78) Xénophon, Anabase II, 3, 1-9 et Diodore, XIV, 26,3-4: de toute évidence, cette ambassade a moins d'importance que la première.

(79) Brown, p. 13, est aussi de cet avis.

(80) Un bon résumé des faits est donné par A.T. Olmstead, op. cil., p. 378379 ; voir aussi Diodore, XIV, 39, 1-4, mais sans évocation de Citésias.

(81) F Gr Hist, T7d ou F32 = Art. 21, 1-5.

(82) F Gr Hist, T7 c ou F30 $=44$ b $20-39$.

(83) C.f. les analyses de Jacoby (col. 2034-2036), de Brown (p. 13-16) et surtout de Lanzani (p. 324-332).

(84) Date donnée par A.T. Olmstead, op. cil., p. 378.

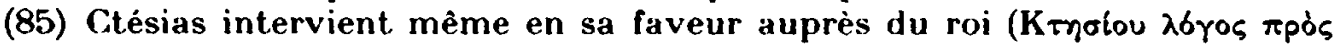

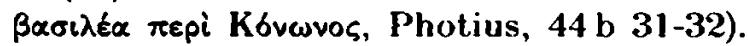


à Salamine de Chypre auprès d'Évagoras ${ }^{86}$ depuis Aigos Potamos, d'autre part aux Spartiates à Lacédémone. Plutarque relève avec malignité que Ctésias rajouta sur une lettre de Conon au Grand Roi un paragraphe où il se mettait lui-même en valeur ${ }^{87}$.

A cet épisode se rattache directement un voyage qui marque le début d'une nouvelle étape dans la vie de Ctésias.

La dernière période de sa vie : Chide, Sparte, Rhodes.

La dernière période de la vie de Ctésias nous est connue par le seul Photius et demeure obscure :

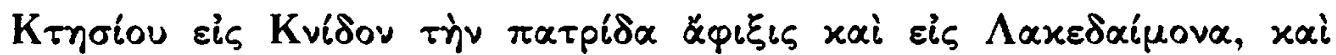

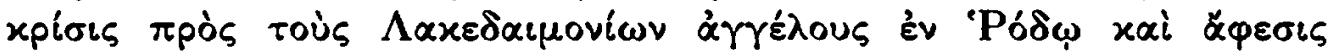
(44 b $39-42=F$ Gr Hist, T 7c).

Ce passage, qui constitue la note presque finale des Persica, a fait l'objet de lectures tout à fait différentes; évoquons d'abord la ponctuation proposée par Estienne ${ }^{88}$ :

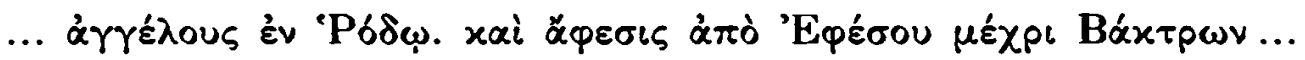

Il rattache $x \alpha \grave{a} \alpha$ $\varphi \varepsilon \sigma \varsigma s$ à la suite du texte qui, sans rapport avec ce qui précède, évoque le compte des relais et des distances d'Éphèse à Bactres et en Inde, du moins d'après les traductions habituelles. Pareille lecture est grammaticalement possible, mais comment Estienne comprend-il le texte? Je suppose qu'il faut voir un départ (de Ctésias) d'Éphèse vers Bactres. On éliminera toutefois cette lecture, certes sans argumentation

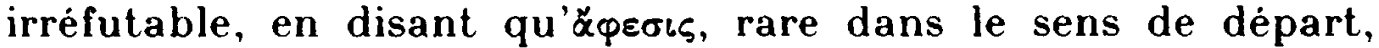
signifie ici acquittement vu la proximité de xpicts (jugement, procès), et en s'appuyant sur la tradition philologique ${ }^{89}$.

(86) Sur l'amitié entre Evagoras et Conon et sur la convergence de leurs vues. voir Isocrate, Euagoras, 52-57, qui les unit dans un mème éloge.

(87) Art. 21,4: pour Brown, p. 17-18, la source de Plutarque est peut-ètre ici Dinon, qu'il préfère à Ctésias. Lanzani, p. 330, n. 1 et 3 , avait déjà souligné la rivalite qui devait exister entre Dinon, lui aussi auteur de Persica, et son prédécesseur.

(88) P. 354: il n'y a malheureusement ni traduction. ni explication.

(89) Baehr, p. 216. corrige Estienne et critique sa ponctuation : "Quid enim

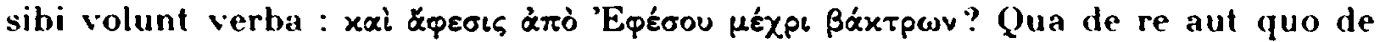
homine ista intelligenda?" Ensuite. Müller. Jacoby (F (ir Hist) et R. Henry (Photius, Bibl., coll. byz., 1959) adoptent la legon de Baehr: König. p. 26. nègligent, ponctue comme Estienne mais traduit à peu près comme les autres. 
Soulignons tout de même qu'une telle ponctuation signifie un retour de Ctésias en Perse et en Orient après l'achèvement de sa mission diplomatique, ce qui, on le verra, n'est pas à exclure totalement.

De quel procès 90 est-il question? Bachr (p. 218) entend par là un procès contre les envoyés lacédémoniens, sans traduire év 'Pó $\delta \omega^{91}$ et sans donner une quelconque explication. Précisons au passage que, pendant les négociations au sujet de Conon, des envoyés de sparte se trouvaient à la cour perse, peut-ètre pas retenus prisonniers mais simplement surveillés ${ }^{92}$. Lanzani (p. 333-334), plus précise, voit un procès fait par les dirigeants spartiates contre leurs ambassadeurs autrefois en poste à Rhodes, puis leur acquittement ; cela se serait déroulé après 395 , date à laquelle les spartiates furent chassés de Hhodes, le chef d'accusation étant peut-ètre que les ambassadeurs n'avaient pas défendu avec assez d’énergie les inlérèts de leur cité à Rhodes. On éliminera également cette interprétation, d’ailleurs unique et sans écho, d'une part parce qu'on comprend très mal comment elle peut sarticuler avec la mention toute proche des ambassadeurs spartiates retenus à la cour perse, dautre part surtout parce quelle oblige Lanzani, qui situe le fait après 395 , à dire que IDiodore commet une erreur en fixant à $398 / 7$ la fin des événements rapportés par les Persica ${ }^{93}$; or, pour des faits aussi problématiques, contester les rares indications données par les textes me paraìt ètre une maladresse méthodologique qui ouvre la porte à un trop grand nombre de spéculations. Bien plutôt, il

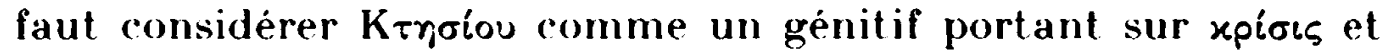
plus précisement, mais sans certitude, comme un génitif objectif $^{94}$ : il y aurait eu un procès à Rhodes contre Ciésias

(9) H. Henry traduit par "differend avec". Cetfe traduction neutre est a mon avis la meilleure.

(91) Comme le remarque, vigilante. I,anzani, p. 33:2. In manuscrit donne év $\lambda \hat{\gamma} \gamma \varphi$.

(92) F Cir IIist. T7 ( $=$ Photius, 4.1 b $35-36$. C'est ainsi que R. Lonis rectifie la

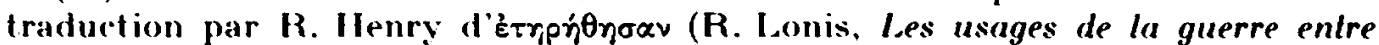
Grecs el Barbares. 1969, p. 69).

(93) Diodore, XIV. 16,6 (cf. infra): I.. Ilolzapfel, art. cit., col. 1270 , remarque déjà qu'une erreur de I lodore est peu probable. I) plus, Ianzani considère ce procès comme lrès secondaire et, voyant que son évocation convient mal à la fin d'un ouvrage, elle est obligée d'avancer l'hypothèse que les Persica seraient inacheves.

(9.4) C"est ainsi que comprennent Jacoby, Brown et R. Henry. In genitif subjectif (pourquoi (atesias aurait-il poursuivi les ambassadeurs?) ne changerait 
intenté par les ambassadeurs spartiates qui revenaient de la cour perse. Reste à trouver, voire à deviner des griefs ${ }^{95}$, mais cela est sans importance. Quant à l'acquittement de Ctésias, il me semble peu probable qu'il ait été dû en partie à l'anneau que lui avait remis Cléarque dans sa prison ${ }^{96}$, vu les réserves émises sur ce personnage (cf. supra, p. 416). Il n'y a lieu ni de dramatiser ni de romancer, et on se contentera de voir là un différend entre

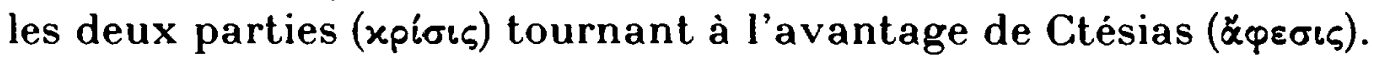

Mais dans quel ordre faut-il lire les indications données par Photius? Quelle succession chronologique doit-on adopter? Brown (p. 18) bâtit le scénario suivant : les envoyés lacédémoniens quittent la cour perse et rentrent chez eux, via Rhodes, où ils arrêtent Ctésias ${ }^{97}$; après un procès (xpíøı), il est acquitté

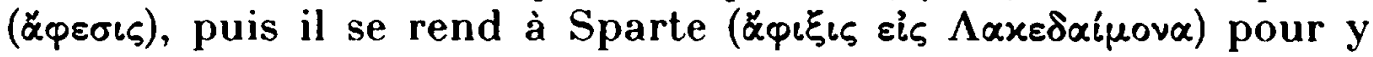
porter la lettre d'Artaxerxès ${ }^{98}$; de là, enfin, il retourne

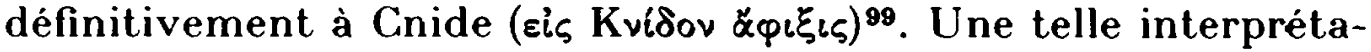
tion est parfaitement cohérente et peut s'harmoniser avec les réalités géographiques. Mais force est de constater qu'elle n'est possible qu'au prix d'un bouleversement dans l'ordre du texte, lu pour ainsi dire à l'envers. Or, celui-ci semble indiquer trois étapes qui se succèdent dans le temps : Cnide, Sparte, Rhodes, et l'on sait que Photius avait lu scrupuleusement Ctésias ${ }^{100}$, apportant beaucoup de soin à rédiger le sommaire d'un ouvrage

pas fondamentalement les choses : il y a procès, litige, différend entre Ctésias et les ambassadeurs.

(95) Brown. p. 18, s'en abstient; pour Jacoby, col. 2036, peut-être les ambassadeurs le rendent-ils responsable d'avoir été retenus si longtemps par les Perses; pourtant, ils ne devaient rien savoir de ce qui se tramait en coulisse. Voir aussi A. Momigliano, art. cit., p. 208-209.

(96) Plutarque, Art. 18,2; Jacoby, col. 2036, voit dans cet anneau presque un passeport pour Sparte : d'après Brown, p. 18, il se pourrait qu'il lui ait sauvè la vie.

(97) Qu'y fait-il? Je pense que, dans ce cas de figure, il revient de sa mission à Chypre.

(98) Cf. supra p. 422 ; d'après Jacoby, col. 2036 , cette mancuvre diplomatique a pour but de donner le change aux Spartiates, alors que le changement de politique des Perses est déjà arrèté.

(99) Votons qu'en parvenant à rentrer dans sa patrie, il réussit là où son lointain prédécesseur Démokédès avait échoué (cf. supra p. 420, n. 68).

(100) C.f. G. Goossens, "L e sommaire des Persica de Ctésias par Photius". Revue belge de Phil. el d'Ilist. 28, 1950, p. $517-519$ ("une ètude très poussée du texte" p. 517). 
lu très vraisemblablement dans l'original ${ }^{101}$. Ce trajet CnideSparte-Rhodes, jamais envisagé, est également plausible : revenant de Chypre, Ctésias fait halte à Cnide, sa patrie (les résidences royales en sont éloignées et il profite de l'occasion), avant d'aller s'acquitter de sa mission à Sparte; de là, sur le chemin du retour, il s'arrête à Rhodes (différend avec les envoyés spartiates). Mais, de Rhodes, où va-t-il s'il va quelque part102? Devant le silence des textes, place est laissée aux conjectures puisqu'il peut tout aussi bien aller à Cinide y finir ses jours ou retourner en Perse ${ }^{103}$; l'enjeu de cette dernière hypothèse est qu'elle confirmerait les tendances pro-achéménides qu'on peut trouver dans les Persica (cf. supra, p. 417-418).

Il y a enfin une troisième manière d'interpréter le passage de Photius, pour peu que l'on examine sa façon de résumer. Son sommaire est en effet très déséquilibré et des passages narratifs assez longs alternent avec des phrases simples d'une extrême sécheresse, ce qui correspond à un traitement inégal des diffèrents livres des Persica ${ }^{104}$. Ici précisément le caractère lapidaire d'une phrase nominale aux éléments simplement

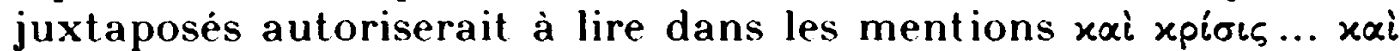

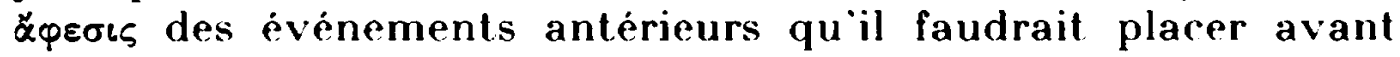

(101) C.f. (;. Coossens, art. cit.: H. Henry, introduction Photius, Bibl., coll. byz., 1959, p. xxiv-xxy; J. M. Bigwood, arl. cit.. Phoenix 30, 1976, p. 2-.4. Je n'ai pas pu disposer de T. Hägg. Photios als l'ermiltler antilier Lileralur. 1975.

(102) Rhodes, encore spartiate en $397 / 6$ quand Clesias doit sy trouver. tombe aux mains des Perses en 396/5 (cf. A. T. Olmstead, op. cit., p. 38:3-38.1). Mais ce changement me parait peu probant pour notre propos.

(103) König (p. 26 n. 13) croit en un retour en Perse de Catésias après ces événements. sappuyant sur $F$ (ir Hist. F27. $71=$ Photius, 44 b 17-19: "La tombe de Cléarque, au bout de huit ans, apparut toute couverte de palmiers que Parysatis, au moment où Cléarque était mort, avait fait planter en secret par sess eunuques" (trad. R. Henry); Ctésias se trouverait donc encore à Babylone en 393/2, ce qui ne serait pas contradictoire (?!) avec la date de 398/7 donnée par Diodore pour la fin des événements contenus dans les Persica. Il me semble inutile de proposer une correction pour ce passage comme le fait Jacoby. col. 2036 ; Brown, p. 6 et 19, a sans doute raison de voir là une information orale fournie plus tard à Catésias par l'un des visiteurs grecs de la tombe et permettant de dater la rédaction des Persica.

(104) C. J. M. Bigwood, art. cit., Phønix 30, 1976, p. 3. G. Goossens, art. cit.. p. 518-20, remarque que les règnes de Xerxès Irr, d'Artaxerxès I'r et de Darius II sont valorisés chez. Photius. ce qui n'était certainement pas le cas chez Ctésias, le proche d'Artaxerxès II ; cette disproportion entre le résumé et l'original apparait clairement dans le sommaire que Photius donne d'un ouvrage' conservé, l'Anabase d'Arrien. 
l'arrivée à Sparte, Photius préférant par souci de brièveté la juxtaposition à la subordination temporelle, évoquant d'abord l'essentiel (Cnide, Sparte) puis l'anecdotique (l'incident de Rhodes). On aboutit ainsi à un trajet Cnide-Rhodes-Sparte ${ }^{105}$ où Ctésias, fort de son acquittement à Rhodes ${ }^{106}$, décide de s'installer pour composer son ouvrage et où il fraternise avec les Spartiates, justifiant par là les critiques que lui adressera Plutarque (cf. supra, p. 415).

Rhodes-Sparte-Cnide, Cnide-Sparte-Rhodes (Perse ?). CnideRhodes-Sparte, tels sont donc les trois itinéraires qu'a pu suivre Ctésias; en préférer un relèverait d'un choix subjectif et paraitrait même assez vain dans la seule optique d'une reconstitution biographique. Mais, puisqu'il est fort probable que Ctésias a écrit ses Persica à un âge assez avancé et dans une retraite définitive, le terme ${ }^{107}$ de son périple importe dans la mesure où il peut déterminer une orientation politique ou partisane de son cuvre historique; en effet, le lieu où un historien élabore son œuvre laisse souvent deviner chez lui des affinités avec le pouvoir en place ${ }^{108}$ et on aurait souhaité dire de Ctésias ce qu'Ed. Delebecque dit de Xénophon : "une partie de son œuvre est écrite à Sparte ou dans une atmosphère lacédémonienne, du point de vue de Sparte et de ses intérêts; mais une autre ne peut se concevoir que rédigée à Athènes, avec la volonté de l'éclairer et de défendre sa cause " ${ }^{109}$. Malheureusement, on a vu qu'on ne peut préciser avec certitude ni l'arrièrefond idéologique de l'œuvre de Ctésias, ni l'endroit où il a rédigé ses Persica ${ }^{110}$ et qu'il est impossible d'éclairer l'un par l'autre.

(105) Le seul possible pour Jacoby, col. 2036, qui ne justifie pas réellement ce choix.

(106) Obtenu non pas grâce à l'anneau de Cléarque (cf. Jacoby. col. 2036) mais parce que, devant remplir une mission à Sparte. Ctésias est un agent diplomatique bénéficiant, à ce titre, de l'immunitè diplomatique généralement respectée par les Grecs comme par les Barbares (cf. R. Lonis, Les usages de la guerre entre Grecs et Barbares, 1969, p. 63-70).

(107) Cnide pour Brown, p. 18-19; la Perse, semble-t-il, pour König, p. 119 ; l'ambiguë "Heimat" pour Jacoby, col. 2036 , où je vois Sparte; simplement la Grèce pour R. Flacelière et E. Chambry, notice Plut., Arl., CUF, 1979, p. 8 et pour C. H. Oldfather, introd. édit. Loeb. Diodore II, réimpr. 1968, p. xxv. Tous les commentateurs ne se prononcent pas sur cette question.

(108) Ainsi, Polybe, favori des Scipion, vante la grandeur de Rome.

(109) Essai sur la vie de Xénophon. 1957, p. 14.

(110) Je me rangerais, mais sans conviction absolue, à l'avis mesuré de Müller, p. 2-3 : "Certe Ctesias in Persidem non rediisse, sed reliquum vitae tempus vel Lacedaemone vel in Cnido patria exegisse videtur." 
Cependant, cette enquête, même si elle est décevante, me semble contribuer à donner à Ctésias un certain crédit et une authentique dimension d'historien; certes, la cohérence de son cuvre n'a pas été démontrée, mais il est vrai que le caractère en général fragmentaire des études sur Ctésias ne favorise pas une perception homogène des Persica. En tout cas, la traditionnelle mise en avant de la fantaisie ${ }^{11}$ de Ctésias, véhiculée à partir de Plutarque ${ }^{212}$, n'est qu'un pis-aller peu satisfaisant, l'inexplicable ne tenant jamais lieu d'explication ${ }^{113}$.

Mais, pour mieux connaître la vie de Ctésias, il reste enfin à s'interroger sur la durée de son séjour en Perse.

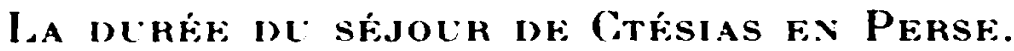

A part les mentions de personnages ou d'événements historiques permettant aisément de dater la vie de Ctésias, les textes ne nous donnent que deux indications chronologiques concernant directement celle-ci. I'une nous renseigne sur l'année sur laquelle s'achèvent les évènements rapportés dans les Persica :

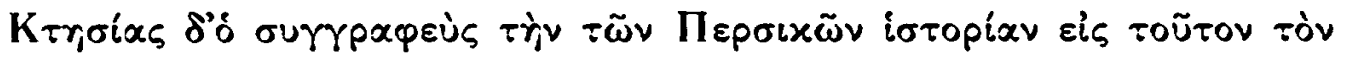

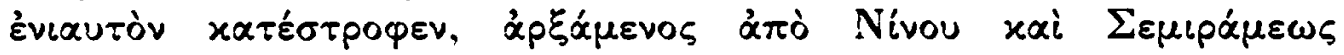
$\left(F\right.$ Gr Hist. T $9=$ Diodore, XIV, 16, 6) ${ }^{114}$.

C'est l'année, à Athènes, de l'archontat d'Ithyclès ${ }^{115}$. l'Olympiade 95,3 , c'est-à-dire $398 / 7$; or. cette date correspond en mème

(111) (:f., entre autres, (). Reverdin, "(irise spirituelle et evasion", Entretiens sur lantiquilé classique. 1 II. Cirecs et Barbares. 1961 (sur Ctésias p. 97-103).

(112) Lanzani. p. 221. semble la seule à remarquer que le jugement de Plutarque sur Ctésias est en réalité très ambigu. En effet. on constate que souvent ses critiques saccompagnent en mème temps dun discret éloge (c.f. 1rt. 1.4:6.9): Plutarque naime ni thomme ni le $\mu \nu \theta \tilde{\omega} \delta \varepsilon s(6,9)$ qu il introduit dans ses récits. mais c'est un bon informateur sachant de quoi il parle: la meilleure preuve en est que, pour la lutte entre Artaxerxès et Cyrus. il s'inspire de Ctésias et non pas de l'Anabase qu'il connait pourtant.

(113) A. Momigliano (art. cit.. p. 183-192) est un des rares à analyser sérieusement la prétendue fantaisie de Ctésias: examinant quelques épisodes de la légende de Vinos et de Sémiramis. l'historien y decouvre non pas une création ex nihilo mais un habile remaniement d'èlements historico-mythiques. le procédé fréquemment utilisé par Ctésias étant celui de la contamination. qui peut porter sur des personnages ou sur des événements.

(11.4) Ciest lunique mention de Chésias au live XIV de Diodore (sur ce live. cf. supra p. 414, n. 29$)$.

(115) Diodore, XIV, 44, 1. 
temps à celle du dernier périple de Ctésias qui, malgré bien des incertitudes, se termine probablement en Grèce comme nous l'avons vu. Ctésias quitte donc sans doute définitivement ${ }^{116}$ la Perse en $398 / 7$ et le fait paraît indubitable ${ }^{117}$.

L'autre indication, à nouveau de Diodore, a suscité bien des commentaires :

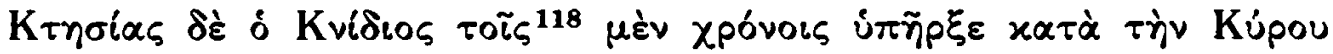

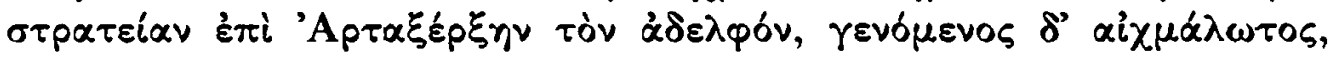

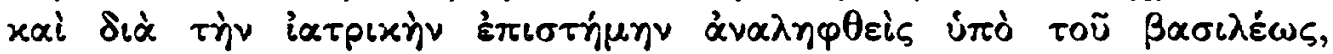

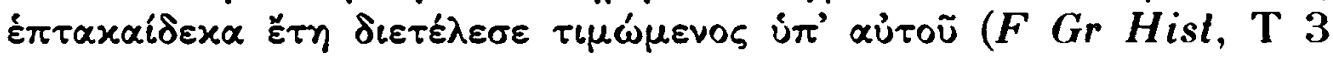
= Diodore, II, 32, 4).

La grande difficulté du passage, que nous traduirons plus loin, provient de la concentration de données hétérogènes qui rendent le texte obscur. Jacoby ${ }^{119}$ y voit peut-être avec raison la combinaison maladroite de deux sources, celle du chronographe

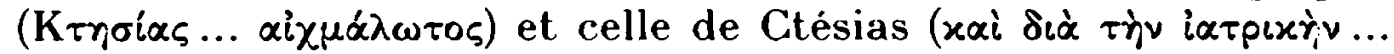

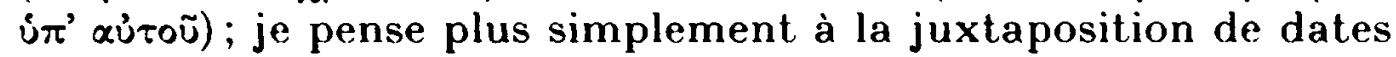
(expédition de Cyrus, capture de Ctésias, engagement comme médecin) et de durées (séjour de dix-sept ans, faveurs du roi), les unes et les autres étant abruptement rapprochées. Passons sur le premier membre de phrase qui pourrait faire croire que Ciésias fut fait prisonnier en 401 à Counaxa : c'est une erreur et envisager pareille hypothèse est en soi une erreur ${ }^{120}$. I se second membre de phrase soulève bien des problèmes. A première lecture, il semblerait que Ctésias ait passé dix-sept ans en Perse au service d'Artaxerxès II; son règne débutant en 405 ou en $104^{121}$, Ctésias aurait donc quitté la Perse en $388 / 7$; or, nous

(116) Sauf pour könig (cf. supra p. 425, n. 103).

(117) C., entre autres. Brown, p. 1 ; Jacoby, col. 2033 , et tous ceux qui se réclament de lui pour la biographie de Ctésias (ainsi, J. M. Bigwood, articles cités. ou R. Drews, The Greek Accounts of Eastern History, 1973, p. 103).

(118) Estienne, p. 386, rajoute ici $x \times \theta^{\prime}{ }^{\circ}$ pódorov: Rettig. p. 10. met cette mention peu adéquate entre parenthèses et Baehr. p. 13. la supprime, suivi en cela par les autres éditeurs. Il s'agit d'un rajout des manuscrits $C$ et $V$.

(119) Col. 2033, critiqué en partie seulement par Brown, p. 4-5.

(1:0) Haehr, p. 13-15, Lanzani, p. 216, Jacoby, col. 2033 et 2035, et König, p. 1. n. 17. estiment que Tretzès (Chil. 1,82-85) et Diodore lui-mème, ici. croient que C. ésias a été capturé à Counaxa: Jacoby surtout réfute cette impossibilité. puisque Plutarque $1 \mathrm{rl} .11,3: 13.3: 14.1)$ et Xénophon (.1nabase 1, 8.26) le citent aux còtés d'Artaxerxès à Counaxa: mais tous ces commentateurs apprécient mal les textes. puisque ni Tzetzès ni Diodore n’évoquent explicitement Counaxa, comme le relève justement Brown. p. 4.

(121) Sur l'imprécision de la date, voir D. M. Lewis, Sparta and Persia. 1977. p. 120, n. 81 . 
savons de source sûre qu'il l'a quittée bien avant, en 398/7. L'incohérence a frappé généralement tous les commentateurs et surtout Müller qui suggère un séjour de sept ans (405/4-398/7) pour faire coïncider les deux dates ${ }^{122}$. Examinons donc cette proposition et les événements qui se déroulaient en 405 et en 404.

En 404, trois cents mercenaires grecs accompagnèrent Cyrus à Babylone où I)arius 1 I se mourait ${ }^{123}$; Ctésias se serait trouvé parmi eux ${ }^{124}$ et le nouveau roi Artaxerxès Il aurait reçu en cadeau le talentueux médecin cnidien de la part de Cyrus qui, accusé de trahison par Tissapherne, se serait ainsi en quelque sorte réconcilié avec son frère. Avoir èté enrôlé par Cyrus puis cédé à Artaxerxès II sans espoir de retour en Grèce aurait fait

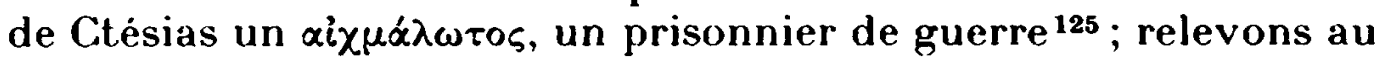
passage que ce $\operatorname{mot}^{126}$ n'est sans doute pas de Ciésias lui-même mais probablement de Diodore qui l'emploie 129 fois ${ }^{127}$ dans son cuvre. Cette reconstitution à partir de faits historiques paraît satisfaisante ${ }^{128}$, et il en va de mème si l'on considère la date de

(122) Il ne semble pas que Müller ait proposé de corriger l'indiscutable

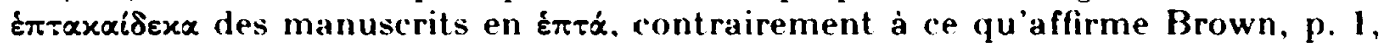
qui se réfère à l'édition de Ctésias par Müller de 1862. A ma connaissance, il n'y a qu'une seule édition de Müller. celle de 1844 (cf. supra. p. 409, n. I), où, sans apporter de correction au texte (cf. p. 11), il suggère très prudemment ceci : " Nam si quidem Artaxerxes rex (souligné) Ctesiam ceperit, fieri id non potuit ante 405 a. chr., ita ut non septemdecim, sed septem tantum annos apud eum (= Art.) degere potuisset (105-398)" (p. 2).

(123) C.f. Xénophon, Anabase I, 1-3, qui passe l'endroit sous silence, mais il s'agit bien de Babylone (cf. F Gr Hist, F16.57= Photius, 43 b 4) comme le confirme A. T. Olmstead, op. cit., p. 371.

(124) Iypothèse de L. Holzapfel, art. cit.. col. 1267 , qui place faussement la scène à Suse: mais c'est Brown, p. $2-3$, qui reconstruit les circonstances de la remise de Cuésias à Artaxerxès. Jacoby, col. 2035, rejette entièrement cette hypothèse : Ctésias, fanfaron, en aurait parlé.

(125) C. Brown, p. 3 ; cela me parait admissible.

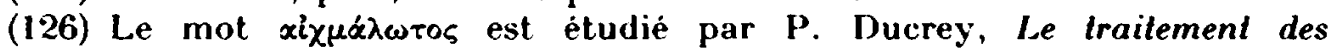
prisonniers de guerre dans la Grèce antique, 1968, p. 16-20; on le trouve seulement à sept reprises chez. Hèrodote, à seize chez. Thucydide et cinq fois dans I'Anabase où les occasions d'évoquer des prisonniers ne manquent pas; l'emploi du mot s'élargit avec le temps et devient très courant chez Diodore, Plutarque et Arrien.

(127) Cf. Lexicon in Diodorum Siculum, ed. J. I. Mc Dougall, 1983.

(128) L'objection de Rettig, p. 12, (Xénophon et Ctésias n'ont pas servi Cyrus ensemble, sinon Xénophon en aurait parlé) n'est pas valable, car Xènophon n'a rejoint Cyrus qu'en 401 (cf. Ed. Delebecque, op. cit., p. 83-85). 
405. Ctèsias, médecin engagé volontaire dans la flotte athénienne, aurait été fait prisonnier à la bataille d'Aigos Potamos par le Spartiate Lysandre qui l'aurait remis à son alliè perse, tout proche, Pharnabaze, ce dernier envoyant à la cour royale et particulièrement à Artaxerxès un de ces médecins grecs tant

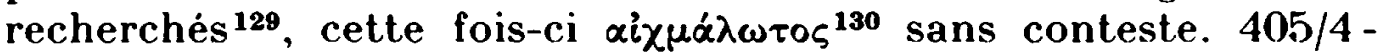
$398 / 7$, telles seraient donc les dates du séjour en Perse de Ctésias $^{131}$, si l'on admet la suggestion de Müller habilement étoffée par les propositions de Brown et si l'on estime que ce séjour coïncide entièrement avec une partie du règne d'Artaxerxès II.

Mais, Diodore donnant $\dot{\varepsilon} \pi \tau x \alpha i \delta \varepsilon \varkappa \alpha$, il est parfaitement possible de croire que Ctésias a effectivement passé dix-sept ans en Perse; il faut alors remonter dans le temps, avant le règne d'Artaxerxès II, pour trouver la date de sa capture et reconnaître qu'il a été fait prisonnier à l'époque de Darius II, précisément en $415 / 14$ si l'on compte à partir de la date bien établie de 398/7. Certes, on se heurte fortement au texte de

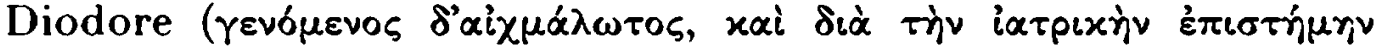

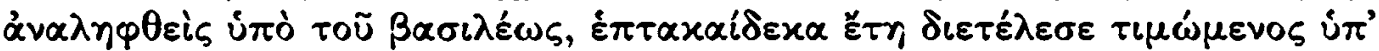

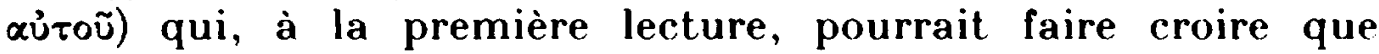
Ctésias, prisonnier sous Artaxerxès, passa dix-sept ans auprès de lui, couvert d'honneurs, ce qui, comme on l'a relevé, est absolument impossible ${ }^{132}$; mais les indications données sont disparates et correspondent à différents moments. Ainsi,

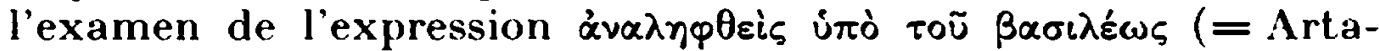

(129) Cf. Brown, p. 1-2, qui réfute aussi d'èventuelles objections.

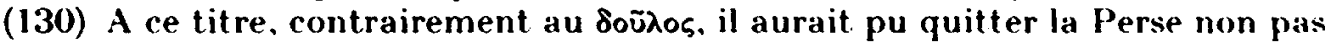
comme il l'a fait mais contre une rançon (cf. R. Lonis, op. cit., p. 51-54).

(131) Cf. R. Henry, Photius, Bibl., coll. byz.. 1959. p. 105, n. 2; R. Drews, op. cit., p. 103 ; J. M. Bigwood, art. cités, Phonix 30, 1976, p. 1, n. 1 et 32. 1978 , p. 19. Ces deux derniers se réclament de Jacoby ; pourtant, il a une position très particulière (col. 2033-35) : tout en reconnaissant la justesse de la suggestion de Müller, il maintient néanmoins vaniteux, exagère délibérément la durée de son séjour en Perse pour marquer sa supériorité sur ses prédécesseurs (Jacoby songe sans doute à Apollonidès de Cos).

(132) Rettig, p. 13-14, qui reconnait qu'il a été capturé sous Darius II, relève justement la difficulté majeure du texte, car Diodore ne dit pas combien d'années Ctèsias a passé en Perse, mais combien d'années il a passé à la cour du roi. Or, $\beta \alpha \sigma i \lambda \varepsilon \omega s$ désignant indiscutablement Artaxerxès II. on a vu qu il est impossible. vu la date de son arrivée au pouvoir, que Ctésias ail passé dix-sept ans auprès de lui. La mention "en Perse" devra nécessairement figurer dans la traduction. 
xerxès II) montre bien qu'il ne s'agit pas du tout de la reprise de

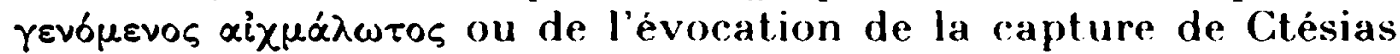
par Artaxerxès; en effel, d'une part, s'appliquant aux prisonniers, $\lambda a \mu b a ́ v e \imath v$ est plus fréquent que ses composés, d'autre part Thucydide surtout l'emploie, et encore dans un tour particulier (la proposition relative), et enfin $\lambda \alpha \mu b a ́ v \varepsilon \iota v$ se fait plus rare dans la langue postérieure où en général il est accompagné de mots

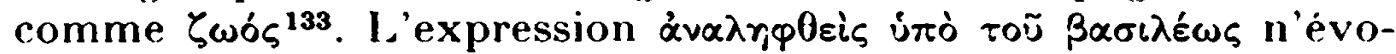
que donc que l'engagement par Artaxerxès de Citésias comme médecin ${ }^{134}$.

Mais que se passait-il aux alentours de 415/14, à l'époque de Darius II, qui puisse expliquer la présence en Perse de Ctésias? Brown date de 413 un événement controversé et difficile à situer ${ }^{135}$ : la révolte du satrape de Lydie Pissouthnès ${ }^{136}$ contre l'autorité royale. On connaît l'épisode grâce à Citésias Photius ${ }^{137}$ : le rebelle Pissouthnès, abandonné en pleine campagne par l'Athénien Lycon et ses mercenaires achetés par l'ennemi, se rend et est emmené devant le roi; celui-ci le fait jeter dans la cendre et récompense le traitre Lycon. Quant à Ctésias, il aurait été au service de Pissouthnès sans faire partie des odieux mercenaires de lycon, et son statut de médecin lui aurait valu la vie sauve ${ }^{138}$; plutòt que de le considérer, avec Brown, comme un volontaire dans les rangs de Pissouthnès, je pense qu'il a été enrôlé dans l'armée de celui qui fut autrefois son vainqueur, bien que cet usage soit beaucoup plus répandu à l'époque hellénistique ${ }^{139}$. Car il aurait été fait prisonnier

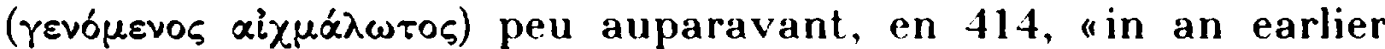
engagement" et, malgré sa qualité, mis au courant des projets de son chef puisqu'il est le seul historien à en faire part ${ }^{140}$.

(133) Cf. P. Ducrey, op. cit., p. 34-36 : on lit aussi l'intéressante remarque suivante : "C’est le participe passif, aoriste ou parfait, du verbe $\lambda \alpha \mu b \& v \omega$ qui semblerait devoir rendre la notion de prisonnier, mais force est de constater que ces formes, bien qu'attestées, ne sont guère répandues" (p. 36).

(134) La traduction donnée par J. I. Mc Dougall dans son lexique de Diodore est tout à fait juste ( $\alpha v \alpha \lambda \alpha \mu 6 \alpha v \varepsilon \iota v$ II, 32,4 "to take with one").

(135) C.f. p. 8-9; cf. aussi D. M. Lewis, op. cit., p. 80-81.

(136) Thucydide le mentionne plusieurs fois (I, 115,4-5; III, 31, I; 34, 2) mais dans des contextes et à des moments tout à fait différents (cf. Brown, p. 8, et D. M. Lewis, op. cit., p. 59-62).

(137) F (ir Hist, F15, $53=$ Photius, 42 b 36-41 et 43 a 1-4.

(138) Cf. Brown, p. 9 ("a strong possibility").

(139) C.f. P. Ducrey, op. cil., p. $101-105$.

(140) C.f. Brown, p. 10. 
Ensuite, ses qualités de médecin l'auraient signalé à l'attention de Tissapherne, le nouveau satrape de Sardes, qui en 404 à Babylone (cf. supra p. 429 et n. 123, évidemment avec des circonstances différentes) l'aurait introduit auprès d'Artaxerxès

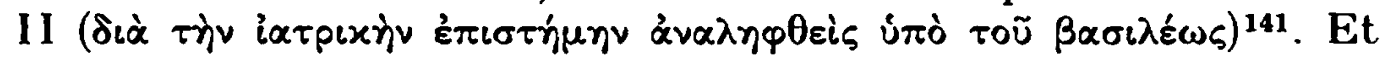
voilà que se dessine, pour mieux répondre à une question déjà posée ${ }^{142}$, l'éventualité d'un second séjour de Ctésias à Babylone.

Je crois donc que Ctésias a probablement séjourné en Perse de $415 / 4$ à $398 / 7$, c'est-à-dire dix-sept ans, et, après bien des détours, on revient à une opinion déjà émise autrefois ${ }^{143}$; en effet, la fin du séjour est datée avec certitude ${ }^{144}$ et le début précède de peu un événement - la révolte du satrape Pissouthnès en 413 - qui, même discuté, est très vraisemblable; surtout, la leçon des manuscrits de Diodore, II, 32,4

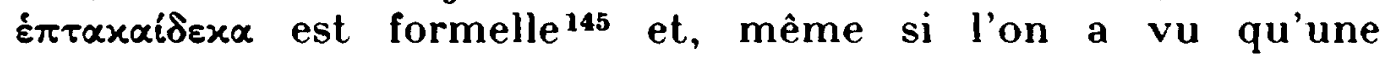
éventuelle correction Ẽ $\tau \tau \alpha$ pourrait aussi se justifier ${ }^{148}$, il n'y a pas lieu de mettre en doute la bonne foi de Ctésias ${ }^{147}$ ou la fidélité de Diodore.

Nous terminons en proposant une traduction du passage de Diodore en précisant les moments et les périodes évoqués :

"Ctésias de Cnide vécut à l'époque de l'expédition de Cyrus contre son frère Artaxerxès (401); prisonnier de guerre (415/4) puis attaché par le roi à son service pour ses connaissances médicales (404, Artaxerxès II), il passa dix-sept ans en Perse (415/4-398/7), honoré de ses faveurs (404-398/7)."

(141) Ibid.

(142) C.f. supra, p. 413-414 et n. 26 p. 413.

(1.43) Baehr, p. 15, propose 416-398, suivi en cela par Lanzani, p. 219: Rettig, p. 9, propose 414-397.

(144) Brown, p. 10, va jusqu'à souligner le caractère réfléchi et prémédité de son départ de Perse, possible à deux conditions : se rendre utile au roi en le quittant (Ctésias y réussit en contribuant à l'engagement de Conon) et se faire remplacer comme médecin. Mais, sur ce dernier point, son hypothèse (le médecin Polycritos de Mendé succédant à Ctésias, p. 17) me parait fragile car elle ne repose que sur une simple mention de l'individu par Plutarque, Art. 21,3.

(145) Elle est confirmée par Tzetzès (Chil. I, 85) qui, peut-ètre, est l'écho d'une tradition manuscrite plus proche de l'archétype de Diodore II que les mss actuellement en notre possession.

(146) C.f. supra, p. 429-430.

(147) Ce que fait Jacoby, col. 2033 (cf. supra p. 430, n. 131). 
Les dates phobahles de la vie de Citésias.

- Entre 451 ? et 441 ? : naissance à Cnide ${ }^{148}$.

- 415/4 : est peut-ètre capturé par le satrape de Sardes Pissouthnès; début de son séjour en Perse qui allait durer dix-sept ans ${ }^{149}$.

- 413: révolte de Pissouthnès; Ctésias peut-être entre les mains du satrape Tissapherne.

- 104: à Babylone, Tissapherne le présente sans doute au nouveau roi; Ctésias devient ainsi le médecin personnel de la famille d'Artaxerxès II

- 401 : à Counaxa, il guérit le roi de sa blessure puis participe aux tractations pour désarmer les mercenaires grecs.

- 399/7 : rôle actif d'intermédiaire dans une opération diplomatique dont l'issue voit l'engagement par les Perses de l'Athénien Conon comme amiral 150. A cet effet, il se rend à Chypre puis (dans quel ordre ? ${ }^{151}$ ) à Cnide, à Sparte et à Rhodes. La période couverte par les Persica s'arrête là.

- 398/7 : retour sans doute définitif 152 en Grèce où Ctésias commence à rédiger les Persica et ses autres ouvrages ${ }^{153}$ (à quel endroit précis?).

(148) Dates proposées par Brown, p. 10 et 19. 441 étant avancé avec: prudence à partir d'une comparaison avec la vie de Xénophon qui serait né en 128 (en 426 cependant pour Ed. Delebecque, op. cil., p. 24). Je ne crois pas qu'on puisie se prononcer avec exactitude (cf. Jacoby, col. 2036).

(149) I.es motifs de son départ de Cnide me semblent difficiles à trouver: pour Lanzani, p. 335, il s'exile volontairement; pour Brown, p. 11, qui interprete abusivement deux courts passages de Galien et d'Oribase $(=F$ Gr Hist, F67 et F68, il s'est joint a quelque expédition militaire dans l'espoir d'épanouir ailleurs ses talents de médecin mal appréciés à Cnide.

(150) I)ates données par A. T. Olnustead, op. cil.. p. 378-380.

(151) C.f. supra p. $422-426$.

(15:2) Sauf pour König (p. 26, n. 13, p. 29, n. 2 et p. 119) qui suppose un retour en Perse et la prèsence de Ctesias à Babylone en 393/2 (cf. supra p. 425, n. 10:3); (Ctésias aurait alors accompli un long et pittoresque voyage alimentant plus tard ses Indica et comparable a l'Anabase de Xénophon (contra, Brown, p. 6). König me parait peu convaincant surtout par ce qu'il maintient la date de $39 \times / 7$ comme fin des événements rapportés par les Persica; or. pourquoi Ctésias aurait-il arrèté là son récit alors qu'un séjour prolongé lui permettait de le poursuive plus en avant?

(15:3) Principalement les Indica (F (ir Hist, F45 à F52); les autres ceuvres

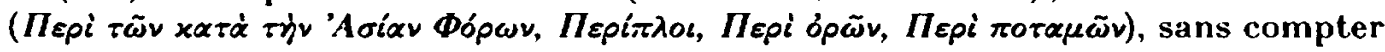


- 393/2 : terminus post quem pour la publication des Persica ${ }^{154}$.

- bien avant 385 : terminus ante quem pour la publication des Persica ${ }^{155}$.

Bernard Eck.

des fragments douteux ou de provenance indéterminée, sont très peu conservées (F Gr Hist, F53 à F74). Pour un bon exposé sur tous ces ouvrages mineurs, voir Jacoby, col. $2037-2040$.

(154) Cf. Brown, p. 6 et 19, d'après les palmiers couvrant la tombe de Cléarque au bout de huit ans, celui-ci ayant été exécuté après Counaxa (cf. supra p. $425, \mathrm{n} .103)$.

(155) Je propose cette date qui est celle de la rédaction de l'Anabase (d'après Ed. Delebecque, op. cit., p. 204-205, avec discussion) car Xénophon avait certainement lu les Persica (cf. supra p. 414). On peut ainsi corriger certaines affirmations insuffisamment fondées (pour J. M. Bigwood, par exemple, il écrivit ses Persica entre 398/7 et autour de 390, "Ctesias as historian of the Persian Wars », Phœnix 32, 1978, p. 19). 